\title{
The Functional Visual Field During Picture Viewing
}

\author{
Walter W. Nelson \\ University of Minnesota (Minneapolis)
}

Geoffrey R. Loftus
University of Washington

In four experiments, pictures of complex, naturalistic scenes were shown, followed by a two-alternative forced-choice recognition test in which the targets and distractors differed in only a single, critical detail. Eye movements were recorded at the time of study in the first two experiments. In Experiment 1 we investigated eye movements during short initial exposure times and found that if the nearest fixation to the critical detail was further than about $2^{\circ}$ of visual angle, performance was no better than chance. Experiment 2 replicated Experiment 1 using longer exposure times and an expanded set of pictures. Performance was still found to decrease with increase in distance of the nearest fixation to the critical detail, but not quite to chance. In Experiments 3 and 4 we controlled where the subject's first fixation occurred using a prefixation point of light. The results indicated that the performance again decreases with increasing distance from the critical detail; however, performance did not fall completely to chance levels. In Experiment 4 a verbal recognition test was included, and overall performance was still slightly better than chance at extreme distances. It was concluded that some information is stored from the visual periphery during picture viewing.

When a person views a static scene, such as a picture, the scan pattern takes the form of short periods of time during which the eye is relatively stationary (fixations) separated by quick jumps of the eye from place to place (saccades). It is generally agreed that visual information processing takes place during fixations, whereas vision is essentially suppressed during saccades.

The reason that the gaze shifts from place to place across the scene is quite simple. The retinal cone receptors, which provide maximum visual acuity, are concentrated in the fovea, a region of the retina subtending a visual angle of only about $2^{\circ}$ horizontal $\times 1^{\circ}$ vertical. Acuity drops off rapidly with increasing retinal distance from the fovea; accordingly, fine detail perception can only be carried out within a rather

This research was supported by National Science Foundation Grants BNS76-23367 and BNS79-06522 to Geoffrey Loftus.

We thank George Bissonnette for assistance in running Experiments 3 and 4.

Requests for reprints should be sent to Geoffrey Loftus, Department of Psychology, University of Washington, Seattle, Washington 98195. limited portion of the visual field. It is for this reason that the point of regard is often considered to be the locus of attention at any given instant (cf. Just \& Carpenter, 1976). Note that "attention" is ipso facto assumed to consist of fine detail encoding when defined in this way.

Despite the importance of foveal processing, several lines of evidence lead to the conclusion that information arriving from the visual periphery is also important for picture perception. First, it has been demonstrated that subjects quickly fixate on informative areas of a picture where "informative" is defined either in terms of subjective ratings (Mackworth \& Morandi, 1967) or in terms of the a priori probability that a particular detail belongs in a particular scene (Loftus \& Mackworth, 1978). For such selective scanning to occur, some peripheral processing is necessary. Second, it has been found that the gist of a picture can be assessed during a single eye fixation. Indirect evidence for this notion comes from Loftus and Mackworth (1978), and it has been demonstrated directly both by Biederman and his colleagues (Biederman, 
1972; Biederman, Rabinowitz, Glass, \& Stacy, 1974) and by Potter (1975). Assessment of gist must certainly require evaluation of visual information arriving from the picture as a whole, that is, of information that comes, for the most part, from the periphery.

We see, then, that peripheral information plays a nontrivial role in the perception of a static scene. But is peripheral information used for anything else? In particular, is it stored in long-term memory, whence it can be used subsequently in, for example, a picture-recognition test? Or is peripheral information transient - is it information that is used merely as an aid in scanning and then discarded? It is to these questions that the four experiments reported in this article are addressed. These experiments all employed a picture-recognition paradigm involving an initial study phase followed by a two-alternative forced-choice test in which target and distractor picture differed only in terms of a single, critical detail (CD). Encoding of the CD during study is therefore necessary for correct performance at test. Of primary interest is the relationship between recognition performance and the distance (in visual angle) of the closest fixation to the CD at study.

\section{Experiment 1}

In the first two experiments, exposure time of the stimuli was varied at study. In Experiment 1, exposure times of 250,500 , and $1,000 \mathrm{msec}$ were chosen to allow roughly one, two, and four fixations per picture, respectively. These numbers of fixations were used so as to keep to a minimum the incidence of multiple fixations on the $\mathrm{CD}$.

\section{Method}

Subjects. Twelve undergraduates at the University of Washington participated in response to a newspaper advertisement. They all had $20-20$ vision, were paid $\$ 3.00$ for participating in the $1-\mathrm{hr}$ experiment, and participated individually.

Stimuli and design. The stimuli consisted of 78 pairs of color slides of rather complex, naturalistic scenes (e.g., backyards, streets, beaches, etc.). Each pair differed only in that an object in one slide was replaced by another object of comparable size in the same location (e.g., a woodpile, next to a building, with a paint roller on top paired with the same woodpile with a baseball bat on top). The typical size of the objects was about $.5^{\circ}$ to $1^{\circ}$ of visual angle, and their positions in the picture were relatively random (e.g., not concentrated in the center). The pictures themselves subtended an angle of about $10^{\circ}$ on the screen.

Subjects viewed 1 member of each of the 78 pairs at study. The three exposure times were randomly ordered with the only constraints being that an equal number of slides appear at each exposure time and that each exposure time be shown before a single exposure time could be repeated. Across subjects, both members of a pair occurred as both target and distractor, thereby resulting in six combinations of exposure time and target/distractor. Two subjects participated in each combination, where exposure time was a within-subjects variable and target/distractor was a between-subjects variable. Presentation and test orders were held constant over all six combinations; however, presentation and test order were randomized with respect to each other.

Apparatus. During study, the target slides were projected by a Kodak random access projector equipped with a Gerbrands tachistoscopic shutter. A second random-access projector was used to project a 500-msec mask that immediately followed the offset of the target slide. The masking slide was constructed so that when the target was presented simultaneously with the mask, no information could be extracted from the target. At test, both projectors were used to present a target and distractor slide, one above the other on the screen. The quality of the two images was indistinguishable. During study, subject's eye movements were recorded using a Polymetric Eye Movement Recorder, Series V-1164. Output of this device consisted of a videotape of the same scene viewed by the subject along with a spot of light that darted about the scene and corresponded to the fixation position (see Loftus, 1979, for a complete description).

Procedure. At study, subjects were informed that they were going to view rather complex, naturalistic color scenes for brief periods of time and that a recognition test would follow the presentation of the slides. They were also instructed that the exposure times would be randomized. Twelve practice slides were presented to familiarize subjects with the study procedure. Immediately after the completion of the study phase, subjects were informed that the pairs of pictures in the two-alternative forced choice differed in a single detail, and subjects were instructed to answer "top" or "bottom," corresponding to whichever picture they remembered seeing before. Subjects were told to guess, if necessary. Subjects were further informed that a random half of the targets would appear on the top and the other half would appear on the bottom (which was indeed true).

\section{Results}

For all dependent variables analyzed in the four experiments, a mean was computed individually for each condition for each subject, and analysis was performed on 
these subject means. Thus, subject selection effects do not enter into any of the results.

The first three columns of Table 1 (first row) indicate probability correct as a function of the distance of the nearest fixation to the $C D$. The distance measure was obtained by watching videotape records of the eye-movement pattern slowed to one-tenth normal speed. The location of each fixation was noted, and the distance of the closest fixation measured from the edge of the CD was recorded. The accuracy of measurement is $\pm .5^{\circ}$ of visual angle. Distance intervals here (and in Experiment 2) were selected so as to yield approximately equal numbers of observations for each interval. A one-way analysis of variance (ANOVA) was performed yielding a significant ${ }^{1} F(2$, $22)=18.4, M S_{\mathrm{e}}=.0146$. The $95 \%$ confidence interval around all three means is shown in the fourth column. It appears that when the subject's nearest fixation to the $C D$ is beyond about $2^{\circ}$ of visual angle, performance drops to, or close to, chance.

Unconditional response probabilities for the 250-, 500-, and 1,000-msec exposure times were $.618, .654$, and .699 , respectively. This increase is significant according to an increasing monotonic trend analysis, $t(22)=2.56, M S_{\mathrm{e}}=.055$, in keeping with the usual finding of a robust exposure time effect (e.g., Loftus \& Kallman, 1979; Potter \& Levy, 1969). The question may now be raised: To what degree is this

Table 1

Recognition Memory Performance (Probability Correct) as a Function of Distance of the Nearest Fixation to the $C D$

\begin{tabular}{|c|c|c|c|c|}
\hline \multirow[b]{2}{*}{ Stimuli } & \multicolumn{3}{|c|}{$\begin{array}{c}\text { Distance to the CD } \\
\text { (degrees of visual angle) }\end{array}$} & \multirow{2}{*}{$\begin{array}{c}95 \% \\
\text { confidence } \\
\text { interval }\end{array}$} \\
\hline & 0 & $.5-1.7$ & 1.8 and up & \\
\hline \multicolumn{5}{|c|}{ Experiment 1} \\
\hline Color & .861 & .628 & .503 & \pm .071 \\
\hline \multicolumn{5}{|c|}{ Experiment 2} \\
\hline $\begin{array}{l}\text { Black and } \\
\text { white } \\
\text { Color }\end{array}$ & $\begin{array}{l}.811 \\
.804\end{array}$ & $\begin{array}{l}.652 \\
.739\end{array}$ & $\begin{array}{l}.612 \\
.606\end{array}$ & $\begin{array}{l} \pm .094 \\
\pm .094\end{array}$ \\
\hline
\end{tabular}

Note. $\mathrm{CD}=$ critical detail.

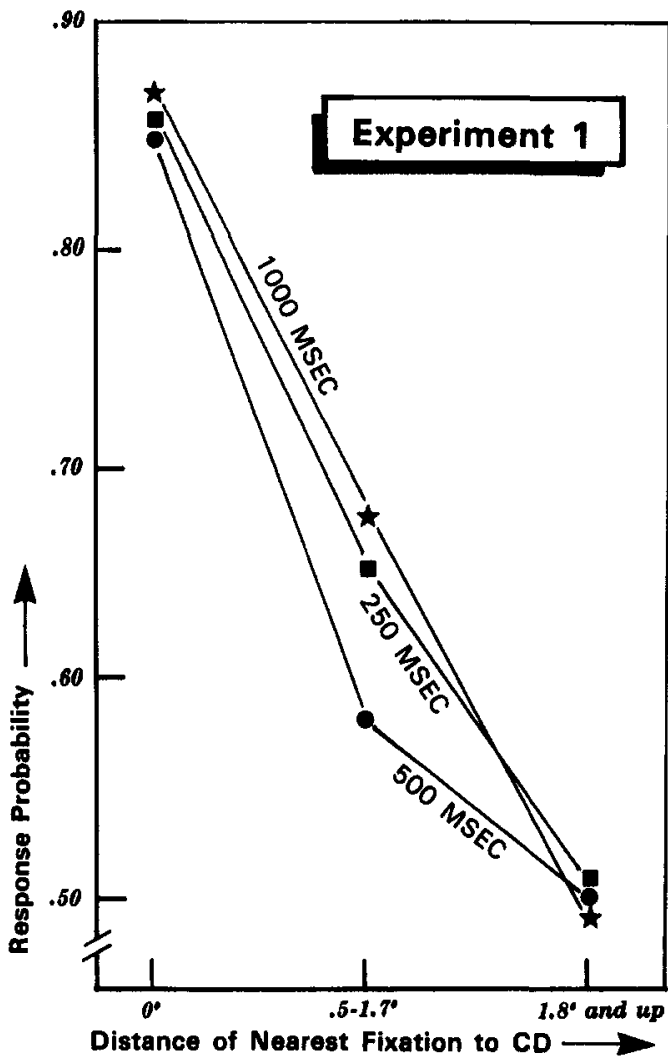

Figure 1. Experiment 1: Probability correct as a function of the distance of the nearest fixation to the critical detail (CD). (Separate curves are drawn for the three exposure times.)

exposure-time effect mediated by the effect of exposure time on the probability of fixating the CD? That is, it might be hypothesized that increasing exposure time simply increases the probability that the CD will be fixated, which in turn is what raises response probability. To investigate this possibility, data from all three exposuretime conditions were further conditionalized on the distance from the CD of the closest fixation to the CD during study. The results of this analysis are shown in Figure 1. The hypothesis just outlined is seen to receive support, as the conditionalized Figure 1 data indicate a minimal effect of exposure time and a large effect of distance. This conclusion is confirmed by a $3 \times 3$, repeated measures ANOVA, which

${ }^{1}$ A significant result refers to significance beyond the .05 level. 
yields a significant $F(2,22)=36.9, M S_{\mathrm{e}}=$ .0335 , for the main effect of distance; a nonsignificant $F(2,22)<1, M S_{\mathrm{e}}=.0231$, for the main effect of exposure time; and a nonsignificant $F(4,44)<1, M S_{\mathrm{e}}=.0224$, for the interaction between exposure time and distance. This result thus indicates that the distance effect mediates the exposuretime effect; that is, increasing exposure time is useful only to the degree that it increases the probability that the CD will be fixated.

\section{Discussion}

The results of Experiment 1 lead to a simple, straightforward conclusion: As the fixation distance from the $C D$ increases, performance decreases, and furthermore, at distances beyond about $1.8^{\circ}$, performance reaches chance (50\%) levels. Thus, a rather specific quantification of the "useful field of view" is suggested by these data, which is about $3.6^{\circ}$ of visual angle.

This conclusion must be tempered by several considerations. First, the accuracy of fixation measurement was, as noted, only $\pm .5^{\circ}$. This means that the distance variable is somewhat noisy relative to the absolute magnitudes of the effects of interest. Second, since the distance effects are correlational, they are open to stimulus selection interpretations. For instance, highly salient CDs may both draw the gaze and render the picture highly recognizable. These two problems will be addressed in Experiments 3 and 4. A third consideration is that the present results are confined to a rather limited range of exposure times (250-1,000 $\mathrm{msec})$. It is not clear what role peripheral vision might play if subjects were allowed a longer time to study each picture. This was the primary reason for conducting Experiment 2, which employed longer exposure times.

\section{Experiment 2}

Experiment 2 differed from Experiment 1 in three ways: Two new exposure times were chosen (750 and 3,000 $\mathrm{msec}$ ), an expanded stimulus set was used, and the exposure times were blocked rather than randomized. The short exposure time was chosen to overlap with the exposure times of Experiment 1, whereas the longer exposure time was chosen to give subjects ample time for multiple fixations on the CD. The color slides from Experiment 1 were used as well as an additional 48 blackand-white slides of the same type of naturalistic scenes as the color slides. The exposure times were blocked in an effort to increase the overall performance on the task.

\section{Method}

Subjects. The subjects were eight undergraduates at the University of Washington who responded to a newspaper ad. Subjects were paid $\$ 3.00$ for participating individually in the 1.5-hr experiment.

Stimuli and design. The stimuli consisted of 80 pairs of color slides - the same 78 pairs used in Experiment 1 -and 2 additional pairs. Forty-eight pairs of black-and-white slides were also used. Each member of a pair served as both target and distractor, resulting in four combinations of exposure time and target/distractor. Two subjects participated in each condition where exposure time was a within-subjects variable and target/distractor was a between-subjects variable. Again, eye movements were recorded during the study phase. All 48 black-and-white slides were presented initially at study followed by the 80 color slides. For four of the eight subjects, the first 24 black-and-white slides as well as the first 40 color slides were shown at the short exposure time, and the last 24 black-and-white and the last 40 color slides were shown at the long exposure time. For the other four subjects, the reverse was true. The blackand-white slides also preceded the color slides in the testing sequence. It should be noted that the blackand-white slides were included only to increase the number of data points per subject. Since color/blackand-white was confounded with study and test order, it was not meant to be a formal independent variable in the experiment.

Procedure. The procedure was identical to that in Experiment 1 except that the subjects were informed that the exposure times would be blocked. Prior to each block, the subjects were informed of the exposure time for that block.

\section{Results}

Separate means were calculated for the black-and-white slides throughout. The second and third rows of Table 1 show the probability correct as a function of the distance to the CD for the black-and-white and color slides, respectively. A test of decreasing monotonic trend indicated a significant distance effect for both black-andwhite, $t(7)=5.28, M S_{\mathrm{e}}=.057$, and for 
color, $t(7)=2.77, M S_{\mathrm{e}}=.030$. However, as indicated by the confidence intervals, performance did not decline to chance at the longer distances. Apparently, useful peripheral information was stored in this experiment. Since there were no significant differences in performance on black-and-white versus color slides, nor were there any interactions involving black-and-white versus color, the results were combined for subsequent analyses.

Unconditional response probabilities were .676 and .770 for exposure times of 750 and $3,000 \mathrm{msec}$, respectively - a difference that was significant, $t(7)=3.58$, $M S_{\mathrm{e}}=.0028$. Figure 2 indicates probability correct conditionalized on the distance of the nearest fixation to the CD. A two-way ANOVA resulted in a significant $F(2,14)=$ $7.08, M S_{\mathrm{e}}=.0158$, for the main effect of

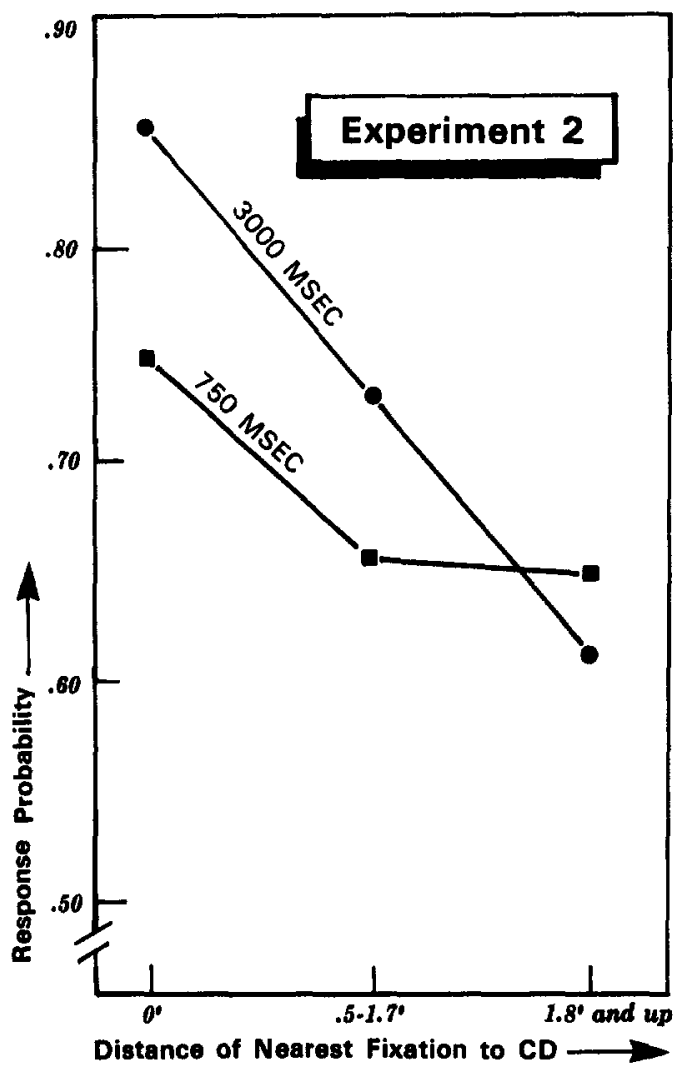

Figure 2. Experiment 2: Probability correct as a function of the nearest fixation to the critical detail (CD). (Separate curves are drawn for the two exposure times.)

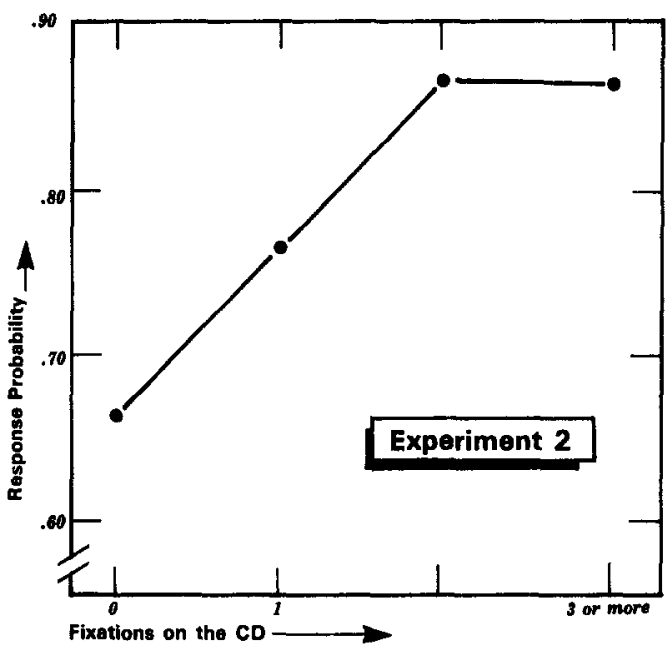

Figure 3. Experiment 2: Probability correct as a function of the number of fixations on the critical detail (CD).

distance; a nonsignificant $F(1,7)=2.94$, $M S_{\mathrm{e}}=.008$, for the main effect of exposure time; and a nonsignificant $F(2,14)=1.97$, $M S_{\mathrm{e}}=.0111$, for the interaction between distance and exposure time. These results indicate that the exposure-time effect is once again mediated, to a large degree, by the distance effect. However, although there is no significant effect of exposure time, 3,000-msec performance does tend to be better than 750 -msec performance.

Figure 3 represents probability correct as a function of the number of fixations on the $C D$. This effect was significant, $F(3,21)=$ $7.74, M S_{\mathrm{e}}=.0094$. In this task it is highly evident that the number of fixations on the $\mathrm{CD}$ is a very good predictor of recognition performance. Performance appears to peak at about three fixations on the $C D$, which apparently are all that is required to successfully encode an adequate representation of the CD.

Table 2 shows performance as a function of the duration of a fixation for those cases in which only one fixation was made directly on the CD. The effect is clearly nonsignificant, $F(3,21)<1, M S_{\mathrm{e}}=.0174$, so that there is no effect of length of fixations on recognition performance. A single fixation, no matter what the duration, is the same as far as performance is concerned. This result is important from a methodological 
Table 2

Probability Correct as a Function of Fixation Duration on the $C D$

\begin{tabular}{|c|c|c|c|c|}
\hline \multicolumn{4}{|c|}{ Duration of fixation on the CD (in msec) } & $\begin{array}{c}95 \% \\
\text { confidence } \\
\text { interval }\end{array}$ \\
\hline $\begin{array}{c}.0-200 \\
.793\end{array}$ & $\begin{array}{c}200-300 \\
.709\end{array}$ & $\begin{array}{c}300-400 \\
.790\end{array}$ & $\begin{array}{c}400 \\
\text { and up } \\
.762\end{array}$ & $\pm \overline{.097}$ \\
\hline
\end{tabular}

Note. $\mathrm{CD}=$ critical detail. Data are included only from instances in which exactly one fixation was made on the $C D$.

standpoint in that what was labeled as one fixation was indeed one fixation and not two fixations in succession in which the saccade was not discernible. ${ }^{2}$ Additionally, this result is in accord with the conclusion reached by Loftus (1972) that a relatively long fixation leads to no better performance than does a relatively short fixation.

\section{Discussion}

The major results of Experiment 1 were replicated with the exception that performance did not fall to chance at long fixation distances. The simplest explanation for this failure is that it is due to sampling error (a possibility that seems viable given the confidence intervals obtained in Experiment 1). An alternative explanation is that subjects can strategically narrow or widen the width of the field from which information can be encoded-and that either knowing the exposure time or having longer exposure times engenders a shift toward widening the useful field of view. In any event, useful information is being obtained in this experiment from a visual field at least as wide as $3.6^{\circ}$.

\section{Experiment 3}

The purpose of this experiment was to allow subjects time for one fixation on each picture and to control where in each picture they fixated. This was to eliminate the effects of subjects selecting where to look in each picture based on the picture's characteristics and thereby to evaluate the itemselection explanation of the distance effect alluded to earlier. An exposure time of
$250 \mathrm{msec}$ was chosen to approximate the duration of one fixation.

\section{Method}

Subjects. Thirty-two undergraduates from the University of Washington participated in groups of four, in return for course credit.

Stimuli and design. The stimuli consisted of the 80 color-slide pairs used in Experiment 2. The exposure time was $250 \mathrm{msec}$ for the study slides, and each study slide was immediately preceded by a $250-\mathrm{msec}$ exposure of a white fixation point. The location of the fixation point was held constant for any given group of subjects to reduce confusion about where to look prior to each picture. A total of four different fixation points were chosen to give a reasonable distribution of distances from the CD. Three of these fixation points were within $2^{\circ}$ of the vertical centerline of the picture, differing only in their vertical positions. The fourth fixation point was approximately $4^{\circ}$ to the left of center. Both members of a pair appeared as target and distractor resulting in eight combinations of fixation point and stimulus slide. Four subjects (one group) served in each of these combinations. A twoalternative forced-choice test was given immediately following the study phase.

Procedure. Subjects were instructed to look at the fixation point prior to each slide and were told that the fixation point would always appear in the same location. A study trial consisted of a $250-\mathrm{msec}$ flash of the white fixation point on the darkened screen followed immediately by the 250 - msec exposure of the study slide. Subjects viewed the 80 study slides and were then tested in a two-alternative forcedchoice test as in Experiments 1 and 2.

\section{Results}

In Table 3 , rows 1 and 2 indicate the means for each of the six distance intervals for three fixation points and separately for the fourth fixation point. Inadvertently, the fourth fixation point selected failed to fall directly on any of the $80 \mathrm{CDs}$, and therefore data are lacking for the $0^{\circ}$ interval for the fourth fixation point. For this reason it was decided not to perform an analysis on these data but to merely treat the entire experiment as a pilot study. It should be noted, however, that the trend of the means does indicate that performance decreases as distance increases. Performance again does not reach chance levels, which indicates that there is still some information being extracted from the periphery even at extreme distances.

\footnotetext{
${ }^{2}$ We thank Tom Cornsweet for noting this as a potential problem.
} 
Table 3

Probability Correct as a Function of Distance From the Fixation Point to the CD

\begin{tabular}{|c|c|c|c|c|c|c|c|}
\hline \multirow[b]{2}{*}{ Fixation point } & \multicolumn{6}{|c|}{ Distance from fixation point to $C D$} & \multirow{2}{*}{$\begin{array}{c}95 \% \\
\text { confidence } \\
\text { interval }\end{array}$} \\
\hline & 0 & $.4-1.0$ & $1.1-1.5$ & $1.6-2.0$ & $2.1-2.5$ & $\begin{array}{c}2.6 \\
\text { and up }\end{array}$ & \\
\hline \multicolumn{8}{|c|}{ Experiment 3} \\
\hline $\begin{array}{c}1-3 \\
4\end{array}$ & $\begin{array}{l}.704 \\
-\end{array}$ & $\begin{array}{l}.690 \\
.625\end{array}$ & $\begin{array}{l}.623 \\
.750\end{array}$ & $\begin{array}{l}.642 \\
.594\end{array}$ & $\begin{array}{l}.610 \\
.704\end{array}$ & $\begin{array}{l}.617 \\
.606\end{array}$ & $\begin{array}{l} \pm .074 \\
\pm .129\end{array}$ \\
\hline \multicolumn{8}{|c|}{ Experiment 4} \\
\hline $\begin{array}{l}\text { Visual test } \\
\text { Verbal test }\end{array}$ & $\begin{array}{l}.794 \\
.650\end{array}$ & $\begin{array}{l}.752 \\
.654\end{array}$ & $\begin{array}{l}.677 \\
.577\end{array}$ & $\begin{array}{l}.686 \\
.562\end{array}$ & $\begin{array}{l}.670 \\
.583\end{array}$ & $\begin{array}{l}.624 \\
.559\end{array}$ & $\begin{array}{l} \pm .050 \\
\pm .050\end{array}$ \\
\hline
\end{tabular}

Note. $\mathrm{CD}=$ critical detail.

\section{Experiment 4}

In addition to being a properly designed version of Experiment 3, Experiment 4 explored the nature of the information that is apparently being extracted during the study phase (especially at long distances from the CD). The method chosen to explore this issue was to replicate Experiment 3 with the additional feature of a two-alternative forced-choice test of the verbal labels corresponding to the CDs prior to the test with the pictures themselves. The rationale was that the use of such a verbal-label test would assess the degree to which verbal information about the CDs is being stored.

\section{Method}

Subjects. Twenty-four University of Washington undergraduates participated in return for course credit. Subjects were run in groups of four.

Stimuli and design. Stimuli and design were identical to Experiment 3 except that prior to the regular test phase with the pictures, a list of 80 pairs of verbal labels corresponding to the 80 target and distractor CDs was given, and subjects were asked to indicate which of the two had occurred in a scene they had viewed during the study phase. The verbal labels were sufficiently detailed to differentiate between the targets and distractors (e.g., "beer can on a lawn" vs. "cigarette pack on a lawn"). Three of the four previous fixation points were used, the one $4^{\circ}$ to the left of centerline being discarded. The six combinations of three fixation points and two values of target/distractor required the six groups of subjects.

\section{Results}

Table 3 (rows 3 and 4) shows the probability correct for the verbal test and the pictorial (or visual) test, both as a function of distance away from the CD of the fixation point. A two-way ANOVA yielded a significant $F(1,23)=63.7, M S_{e}=.012$, for the main effect of verbal versus visual test, and a significant $F(5,115)=7.48, M S_{\mathrm{e}}=.169$, for the main effect of distance. The results of the visual test replicate those of Experiment 3 , indicating that even at extreme fixation points, subjects are able to gain information that is helpful in the subsequent visual test. The verbal test indicates that even though performance is worse than in the visual test, performance is still slightly better than chance at extreme fixation points.

The pattern of the distance function is consistent in both the visual and verbal conditions as well as for Groups 1-3 of Experiment 3: The performance drop is fairly dramatic up to a distance of about $1.5^{\circ}$ and then levels off. Although performance is above chance at greater distances, there is very little, if any, distance effect past $1.5^{\circ}$, at least with the range of distances that it is possible to obtain with the present stimuli.

\section{Discussion}

The performance drop over distance found in Experiments 1 and 2 was replicated in Experiment 4 (and in Experiment 3 as well). Since fixation location was controlled in the latter two experiments, this finding eliminates item selection as an explanation for the effect.

In agreement with Experiment 2, the 
results of Experiments 3 and 4 indicate that even at extreme degrees of visual angle, some small but significant amount of information is being gained in the first fixation. Furthermore, some portion of this information is verbal in nature, or at least it is information that can be used in a verbal test that lacks any sort of visual cues.

The pattern of the function relating performance to distance is interesting and induces the speculation that qualitatively different information is being gathered from the region of $\pm 1.5^{\circ}$ around the fixation point versus the region that is further into the periphery. Such a notion is particularly tantalizing in view of the fact that $\pm 1.5^{\circ}$ corresponds roughly to the foveal region. However, since the function was similar for the visual and verbal tests-which might, on an a priori basis, be thought to test different types of information-this speculation must remain speculative. Additional caution is dictated by the fact that it is rather dangerous to make inferences about the exact, quantitative form of a function based on the limited data of one or two experiments. Nonetheless, it is something to keep in mind with respect to future research.

\section{General Discussion}

\section{Foveal Versus Peripheral Information}

In all four experiments, recognition performance was found to be a strongly decreasing function of the distance between the $\mathrm{CD}$ and the nearest fixation to the $\mathrm{CD}$. The procedure used in Experiments 1 and 2 to explore this function enjoyed some degree of ecological validity in the sense that subjects were permitted to look around the picture in accord with their preferred patterns. In Experiments 3 and 4, only one fixation was permitted, and distance to the CD was experimentally controlled, thereby eliminating item-selection effects. It is comforting that similar results were found with the two procedures.

The results suggest that the distance function takes the form of a sharp decrease up to $\pm 1.5-1.8^{\circ}$ of visual angle and then levels off. In Experiment 1, the leveling off was at chance performance, whereas in Experiments 2-4, above-chance performance was found at these distances. The net result is the conclusion that subjects can store information arriving from the visual periphery. However, it leaves open questions as to the circumstances under which such information is stored. The simplest conclusion is that some small amount of peripheral information is always stored and that the chance performance seen in Experiment 1 is due to sampling error; that is, it simply reflects a lack of statistical power. Another possibility is that the width of the functional visual field is to some degree under the control of the subject and that minor methodological variations across the four experiments resulted in such shifts. Indirect support for such a notion is contributed by the results of Mackworth (1976), which demonstrate that the width of the functional visual field is highly influenced by stimulus factors.

\section{The Nature of Information Acquired Within a Fixation}

There has been a substantial amount of interest in the nature of the information that is acquired at varying distances into the visual periphery. Loftus and Mackworth (1978) have argued that at least some degree of object pattern recognition is carried out for objects as far as $5^{\circ}$ into the visual periphery. In the domain of reading, McConkie and Raynor and their colleagues (e.g., Raynor, McConkie, \& Zola, in press) have been charting the types of information acquired from peripherally viewed words during the reading of text. They have found that under certain circumstances, information is acquired about the individual letters of a word from up to $\pm 5^{\circ}$ into the periphery.

It would appear that the notion of limiting the area from which information is available to that of the foveal region only holds in very restricted cases (e.g., short exposure times, highly specific detail information, and complex pictorial material). In general, we must relax the boundaries of the functional field of view when subjects are given ample time to scan a complex picture. Peripheral 
information has been found to be useful in lower levels of processing, such as in guidance and decision processes used in planning future fixations. Parker (1978) noted that the acuity of the periphery is sufficiently well-defined to be useful in most everyday visual encoding and points out that direct fixations may be necessary only when a high degree of detail information is required. The present study would extend this concept to include such fine-detail analysis as identifying single objects in complex, naturalistic scenes.

The present Experiment 4 indicates that some information from as far as $\pm 2.6^{\circ}$ in the visual periphery is both acquired and stored; and furthermore, this information is of a nature that will allow correct performance on a verbal test. We should like to point out that the absolute difference between visual and verbal performance is of little consequence, since this difference could probably be manipulated at will by appropriate choice of CDs. What is interesting is that the same general distance function is shown by both tests, and the information necessary for both types of tests exists in memory, even when it comes from the periphery. This indicates that at some pointeither at the time of original viewing or at test - the information acquired and stored from the periphery is of sufficient quality that it can be and is used to generate a correct verbal label.

\section{Eye Fixations and Picture Recognition}

Loftus (1972) has demonstrated that the effects on picture-recognition performance of two independent variables-exposure time and incentive-are mediated by their effects on eye fixations; that is, longer exposure time and more incentive are only beneficial insofar as they lead to more fixations on the picture. In Experiments 1 and 2 of the present study, we have carried this analysis one step further: We have found that exposure time is mediated by distance to the CD. That is, it appears that although more exposure time is beneficial only to the degree that it allows more fixa- tions, more fixations are beneficial only to the degree that they increase the probability that a fixation on or close to the CD will be made. Admittedly, these experiments are structured so as to make the CD critical for performance. But in a more general sense, evidence is provided that eye fixations are useful in remembering pictures because the more fixations there are, the greater the likelihood that features will be acquired that will ultimately prove useful for distinguishing a target from distractors.

\section{References}

Biederman, I. Perceiving real world scenes. Science, $1972,177,77-80$.

Biederman, I., Rabinowitz, J. C., Glass, A. L., \& Stacy, E. W., Jr. On the information extracted from a glance at a scene. Journal of Experimental Psychology, 1974, 103, 597-600.

Just, M., \& Carpenter, P. Eye fixations and cognitive processes. Cognitive Psychology, 1976, 8, 441-480.

Loftus, G. R. Eye fixations and recognition memory for pictures. Cognitive Psychology, 1972, 3, 525551.

Loftus, G. R. On-line eye movement recorders: The good, the bad, and the ugly. Behavior Research Methods \& Instrumentation, 1979, 11, 188-191.

Loftus, G. R., \& Kallman, H. J. Encoding and use of detail information in picture recognition. Journal of Experimental Psychology: Human Learning and Memory, 1979, 5, 197-211.

Loftus, G. R., \& Mackworth, N. H. Cognitive determinants of fixation location during picture viewing. Journal of Experimental Psychology: Human Perception and Performance, 1978, 4, 565-572.

Mackworth, N. H. Stimulus density limits the useful field of view. In R. A. Monty \& J. W. Senders (Eds.), Eye movements and psychological processes. Hillsdale, N.J.: Erlbaum, 1976.

Mackworth, N. H., \& Morandi, A. J. The gaze selects informative details within pictures. Perception and Psychophysiology, 1967, 2, 547-552.

Parker, R. E. Picture processing during recognition. Journal of Experimental Psychology: Human Perception and Performance, 1978, 4, 284-293.

Potter, M. C. Meaning in visual search. Science, 1975, 187, 965-966.

Potter, M. C., \& Levy, E. I. Recognition memory for a rapid sequence of pictures. Journal of Experimental Psychology, 1969, 81, 10-15.

Raynor, K., McConkie, G., \& Zola, D. Cognitive Psychology, in press.

Received October 11, 1979 Revision received February 4, 1980 